\title{
The Expressive and the Receptive One Word Picture Vocabulary Test (EOWPVT \& ROWPVT). (A combine pilot study in High School aged children \& data for expressive and receptive language for this population)
}

\author{
Dionisios Tafiadis ${ }^{1,2^{*}}$, Euaggelia Bogindrouka ${ }^{1}$, Kaliopi Kentioglou ${ }^{1}$, Emmanouil Pantazis $^{1}$, Maria Zizika ${ }^{3}$, \\ Maria Tafiadi ${ }^{3}$, Aggelos Papadopoulos ${ }^{3}$, Pagona(Nonika) Koutsogianni ${ }^{3}$
}

From $1^{\text {st }}$ International Congress on Neurobiology and Clinical Psychopharmacology and European Psychiatric Association Conference on Treatment Guidance

Thessaloniki, Greece. 19-22 November 2009

\section{Background}

The present pilot study was the adaption of receptive and expressive language tests' (ROWPVT and EOWPVT) for Greek children aged from 14 years till 18 years and 11 months, and to locate any differences between receptive and expressive language.

\section{Materials and methods}

The commercial versions of the tests were adapted in Greek language by a linguist, three speech language therapists and 2 native speakers of Greek language, having proficiency in English, and two native speakers of English having proficiency in Greek, and changes were contacted, for the best representation of the Greek version. In this research took part 400 participants (m: 200, f: 200) recruited from Greek High Schools. The sample was independent from origin and socio - economic situations. Children with medical problems (ENT, neurological or psychiatric) excluded, because it will influence the test results.

\section{Results}

Statistical analysis of the data revealed that the results obtained are generally consistent to other results reported. No statistically significant differences were found according to sex. Also reliability and validity test were contacted and showed high criterion (a - Chronbach $=.801, \& .819)$.

\section{Conclusions}

The test appears to be sensitive for high school aged Greek population and presents satisfactory criterion, internal consistency, temporal stability, interrater reliability, high content validity. The participants demonstrated clear patterns of responses and there were no differences between expressive and receptive language.

\section{Author details}

'Department of Speech and Language Therapy, T.E.I. of Epirus, loannina, Ioanninon, Greece. ${ }^{2}$ Department of Neural Sciences and Sensory Organs (School of Medicine), University of loannina, Ioannina, Ioanninon, Greece. ${ }^{3}$ Department of Mathematics, National and Kapodistrian University of Athens, Athens, Attika, Greece.

Published: 22 April 2010

\section{doi:10.1186/1744-859X-9-S1-S119}

Cite this article as: Tafiadis et al:: The Expressive and the Receptive One Word Picture Vocabulary Test (EOWPVT \& ROWPVT). (A combine pilot study in High School aged children \& data for expressive and receptive language for this population). Annals of General Psychiatry 2010

9(Suppl 1):S119. 